\title{
Kinetic analysis of the growth rate of sporadic and hereditary medullary thyroid carcinoma: comparing the postoperative calcitonin-doubling rate with the hypothetical preoperative tumor volume- doubling rate
}

Minoru Kihara* (1D, Akira Miyauchi, Hiroo Masuoka, Takuya Higashiyama, Yasuhiro Ito and Akihiro Miya

\begin{abstract}
Background: Our previous kinetic analyses of changes in the tumor volume (TV) of papillary thyroid microcarcinomas during active surveillance revealed that the tumors' growth varied over time from rather rapid growth to shrinkage and that the hypothetical TV-doubling rates (DRs) before the patients' presentation were much larger than their observed TV-DRs, indicating that rapid growth phases preceded their presentation. Whether this phenomenon also occurs in medullary thyroid carcinoma (MTC) was unknown.

Methods: We retrospectively analyzed the cases of 46 MTC patients (18 hereditary, 28 sporadic; 9-80 years old at surgery, median 53.5 years; 19 males and 27 females) with elevated postoperative calcitonin (Ct) measured with the electrochemiluminescence immunoassay suggesting persistent disease. We calculated each patient's Ct-DR and his/her hypothetical TV-DR, using the tumor size and age at surgery.

Results: Ct-DRs (/year) after surgery were $>0.5,0.1-0.5,-0.1-0.1$, and $<-0.1$ in 9, 21, 12, and 4 patients, respectively (median 0.17). The hypothetical TV-DRs (/year) before surgery were $>1,0.5-1.0,0.1-0.5$ and $<0.1$ in $11,21,14$, and 0 patients, respectively (median 0.60). The hypothetical TV-DR was higher than the observed Ct-DR in 41 of the 46 MTC patients and all 18 patients with hereditary MTC, suggesting that a rapid growth phase preceded surgery in these patients.
\end{abstract}

Conclusions: In this series of MTC patients, the pre-surgery calculated hypothetical TV-DRs were significantly higher than the Ct-DRs observed post-surgery, suggesting that there were rapid growth periods before surgery in the vast majority of these MTC patients.

Keywords: Kinetic analysis of the growth rate, Doubling rate, Medullary thyroid carcinoma, Calcitonin, Tumor volume

* Correspondence: kihara@kuma-h.or.jp

Department of Surgery, Kuma Hospital, 8-2-35 Shimoyamate-dori, Chuo-ku,

Kobe, Hyogo 650-0011, Japan

(c) The Author(s). 2020 Open Access This article is licensed under a Creative Commons Attribution 4.0 International License, which permits use, sharing, adaptation, distribution and reproduction in any medium or format, as long as you give appropriate credit to the original author(s) and the source, provide a link to the Creative Commons licence, and indicate if changes were made. The images or other third party material in this article are included in the article's Creative Commons licence, unless indicated otherwise in a credit line to the material. If material is not included in the article's Creative Commons licence and your intended use is not permitted by statutory regulation or exceeds the permitted use, you will need to obtain permission directly from the copyright holder. To view a copy of this licence, visit http://creativecommons.org/licenses/by/4.0/. The Creative Commons Public Domain Dedication waiver (http://creativecommons.org/publicdomain/zero/1.0/) applies to the data made available in this article, unless otherwise stated in a credit line to the data. 


\section{Introduction}

Medullary thyroid carcinoma (MTC) is an uncommon malignant tumor derived from the $\mathrm{C}$ cells (calcitoninproducing cells) of the thyroid gland, and it presents in a sporadic or hereditary variant. Serum calcitonin $(\mathrm{Ct})$ is a very sensitive and specific tumor marker for MTC. In 1984, Miyauchi et al. found that in patients with MTC who had elevated serum $\mathrm{Ct}$ values postoperatively, the serum $\mathrm{Ct}$ values changed exponentially; they reported that the Ct-doubling time (Ct-DT) was a strong prognostic factor for MTC [1]. Twenty-one years later, Barbet et al. performed multivariate analyses on possible prognostic factors for MTC and found that among various clinicopathological factors, the Ct-DT remained an independent prognostic factor [2]. Some of their patients showed a decrease in their serum $\mathrm{Ct}$ values over time, rendering negative values for their Ct-DTs. This created a discontinuity problem among positive and negative $\mathrm{Ct}$ DT values. Barbet et al. were very smart in solving this problem: They converted the Ct-DT to $1 / \mathrm{Ct}-\mathrm{DT}$ for statistical analyses. We recently encountered a similar problem in our kinetic study of tumor volumes during the active surveillance of patients with low-risk papillary thyroid microcarcinomas, since some of these patients also showed decreases in their tumor volumes over time. We converted the tumor volume-doubling time (TV-DT) to its inverse (i.e., 1/TV-DT), and we proposed that this value be called the 'doubling rate (DR)', because it is designed to indicate the number of doublings (or halvings) that occur per unit of time [3]. With the TV-DR we were able to determine the tumor volume kinetics during active surveillance in individual patients in a single figure [3].

For the investigation of tumors' growth before the patients' clinical presentation, we calculated the hypothetical TV-DR - which is an estimate of the tumor growth rate before presentation - by using the patient's age and the tumor size at presentation, assuming that a single $10-\mu \mathrm{m}$ cancer cell was present at the patient's birth and grew at a constant rate. If a tumor arose later than the birth time point, the tumor's growth rate should be higher than this estimate. If the tumor's growth was not constant and there was a slow-growth phase, there should be a period of more rapid growth for the cancer cell to grow to the size observed at presentation. Therefore, the hypothetical TV-DR is the lowest tumor growth rate before presentation.

Our research revealed that in most of the patients, the hypothetical TV-DR was much larger than the TV-DR observed during active surveillance, suggesting that the tumors' growth before the patients' presentation had been much more rapid. In other words, the papillary microcarcinomas had undergone a spontaneous decrease in their tumor growth rates. Some of the tumors even shrank after their presentation. Whether a similar phenomenon also occurs in MTCs has been unknown, and we conducted the present study to investigate the natural history of MTCs before presentation by comparing the Ct-DRs observed after surgery with the hypothetical TV-DRs that were estimated before surgery.

\section{Materials and methods \\ Subjects}

From January 2005 to December 2018, 199 patients underwent their initial surgery for MTC at our hospital. Of these, 46 patients (23\%) had postoperative $\mathrm{Ct}$ values $\geq 10 \mathrm{pg} / \mathrm{ml}$ measured with the electrochemiluminescence immunoassay, suggesting biochemically persistent disease, and they were enrolled in this study (Table 1). They were 19 males and 27 females, ages 9-80 years old at surgery (median 53.5 years). The maximum diameter of the patients' largest tumors ranged from $5 \mathrm{~mm}$ to 75 $\mathrm{mm}$ (median $20 \mathrm{~mm}$ ). All patients underwent a germline RET mutation analysis preoperatively. Twenty-eight patients had no RET gene mutations. The other 18 patients had the RET gene mutations that are regarded as posing the highest risk ( $n=3$ patients), high risk $(n=7)$ and moderate risk $(n=8)$ according to the newest American Thyroid Association (ATA) guidelines [4]. None of the patients underwent any additional surgery, tyrosine-kinase inhibitor therapy, or external beam radiotherapy after the initial surgery. None of the patients had hyperparathyroidism, hepatic cirrhosis, or renal insufficiency. None were treated with a drug that increases calcitonin secretion (e.g., omeprazole, beta-blockers, and glucocorticoids).

\section{Calcitonin assay}

In Japan, calcitonin was measured before April 2015 by using the solid two-site immunoradiometric assay. After that point, calcitonin has been measured by an electrochemiluminescence immunoassay (ECLIA). In the present study, we used only the $\mathrm{Ct}$ values measured with an ECLIA. The patients' Ct values were measured by a laboratory (SRL Co., Tokyo) using the Elecsys ${ }^{\circledR}$ Calcitonin test system (Roche Diagnostics, Tokyo). According to the manufacturer, the normal ranges of basal serum calcitonin are $\leq 9.52 \mathrm{pg} / \mathrm{mL}$ for males and $\leq 6.40 \mathrm{pg} / \mathrm{mL}$ for females, with a lower detection limit of quantification of $0.5 \mathrm{pg} / \mathrm{mL}$.

\section{Measurement of serum $\mathrm{Ct}$ and calculation of the Ct-DR}

The patients' post-operative serum Ct was measured two to four times per year. A median number of total $\mathrm{Ct}$ measurements was 9, ranging from 4 to 10 in this study (Table 1). We calculated each patient's Ct-DR as described [3]. This calculation is easily performed using the Doubling Time, Doubling Rate \& Progression 
Table 1 Clinicopathological characteristics in hereditary MTC patients and in sporadic MTC patients

\begin{tabular}{|c|c|c|c|c|}
\hline Variable & Total & Sporadic MTC & Hereditary MTC & $p$-value \\
\hline No. of patients & 46 & 28 & 18 & \\
\hline Age at surgery, yrs. ${ }^{a}$ & $53(9-80)$ & $59(25-80)$ & $28(9-59)$ & $<0.001$ \\
\hline \multicolumn{5}{|l|}{ Sex, no. (\%) } \\
\hline Male & 19 & 14 & 5 & \multirow[t]{2}{*}{0.220} \\
\hline Female & 27 & 14 & 13 & \\
\hline Max. tumor size at surgery, $\mathrm{mm}^{\mathrm{a}}$ & $20(5-75)$ & $20(9-63)$ & $21(5-75)$ & 0.991 \\
\hline \multicolumn{5}{|l|}{ Extrathyroid extension: ${ }^{b}$} \\
\hline Yes & 2 & 1 & 1 & \multirow[t]{2}{*}{0.999} \\
\hline No & 46 & 27 & 17 & \\
\hline \multicolumn{5}{|l|}{ Ki67 labeling index: } \\
\hline$<5 \%$ & 39 & 25 & 14 & \multirow[t]{4}{*}{0.163} \\
\hline $5-10 \%$ & 2 & 0 & 2 & \\
\hline$>10 \%$ & 4 & 3 & 1 & \\
\hline Unknown & 1 & 0 & 1 & \\
\hline \multicolumn{5}{|l|}{ pN (8th UICC/AJCC staging system): } \\
\hline pNO & 3 & 1 & 2 & \multirow[t]{3}{*}{0.594} \\
\hline pN1a & 3 & 2 & 1 & \\
\hline $\mathrm{pN} 1 \mathrm{~b}$ & 40 & 25 & 15 & \\
\hline \multicolumn{5}{|c|}{ Stage (8th UICC/AJCC staging system): } \\
\hline । & 3 & 1 & 2 & \multirow[t]{3}{*}{0.594} \\
\hline III & 3 & 2 & 1 & \\
\hline IVA & 40 & 25 & 15 & \\
\hline Ct-DR, per year: ${ }^{\mathrm{a}}$ & $0.17(-1.25-5.70)$ & $0.17(-0.42-5.70)$ & $0.21(-1.25-1.90)$ & 0.753 \\
\hline hTV-DR, per yr ${ }^{a}$ & $0.60(0.26-3.65)$ & $0.50(0.26-1.10)$ & $1.21(0.50-3.65)$ & $<0.001$ \\
\hline Observation period, yrs & $4.6(1.0-4.6)$ & $4.6(1.0-4.6)$ & $4.6(1.0-4.6)$ & 0.079 \\
\hline No. of Ct measurements & $9(4-10)$ & $7(5-10)$ & $9(4-9)$ & 0.018 \\
\hline
\end{tabular}

$C t-D R$ calcitonin doubling rate, $h T V-D R$ hypothetical tumor-volume doubling rate, MTC medullary thyroid carcinoma

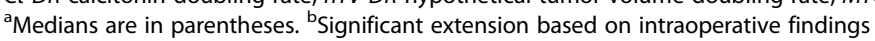

Calculator (freely available from: http://www.kuma-h.or. jp/english/).

\section{Hypothetical tumor-volume doubling rate (hTV-DR)}

We calculated the hypothetical (h)TV-DRs by using the tumor size and the patient's age at surgery, assuming that a single $10-\mu \mathrm{m}$ cancer cell was present at the patient's birth and grew at a constant rate [3]. Tumor diameters were measured with the use of ultrasonography shortly before the patients' surgeries. The maximum diameter (D1) and the diameter in the direction perpendicular to the maximum diameter (D2) were measured. The tumor depth value was often not reliable because of ultrasound shadowing. The volume of each tumor at surgery was calculated using the ellipsoid equation: $\pi /$ $6 \times \mathrm{D} 1 \times \mathrm{D} 2^{2}$ [5]. 'Time' is the time interval between the patient's birth and surgery, i.e., the age (years) at surgery. The hTV-DR was calculated using the Doubling Time, Doubling Rate \& Progression Calculator.

\section{Statistical analysis}

The statistical tests used to compare the differences between the groups were the chi-square test for independence, the Mann-Whitney U-test for skewed variables, and the Wilcoxon signed-rank test for paired skewed variables. A $p$-value $<0.05$ was regarded as significant. All analyses were performed using StatFlex 6.0 software (Artech, Osaka, Japan).

\section{Results}

Table 1 summarizes the results of our comparison of the demographic and clinicopathological features of the patients with sporadic and hereditary MTC. The median age at surgery of the patients with hereditary MTC was significantly lower than the ages of the patients with sporadic MTC $(p<0.001)$. There was no significant difference in sex, max. Tumor size, extrathyroid extension, Ki67 labeling index, pN, or TNM stage between the sporadic MTC patients and the hereditary MTC 


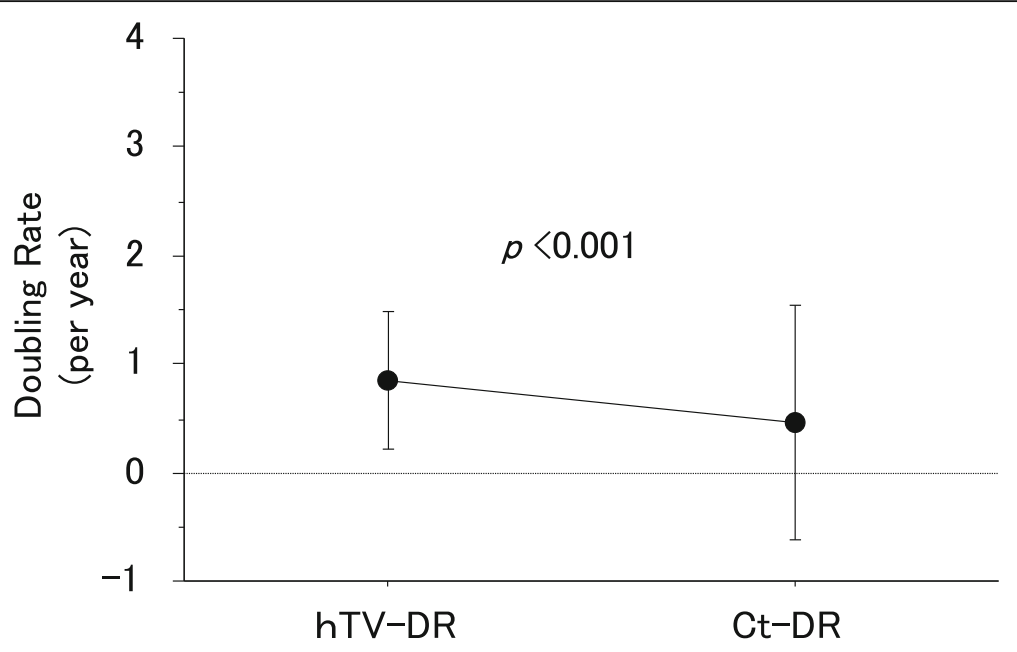

Fig. 1 The calcitonin (Ct)-DR and the hypothetical tumor-volume doubling rate (hTV-DR) values of the 46 MTC patients

patients, both groups having biochemically persistent disease postoperatively.

The Ct-DRs (/year) after surgery in the 46 patients ranged from -1.25 to 5.70 with a median of 0.17 . The hTV-DR values (/year) before surgery ranged from 0.26 to 3.65 with a median of 0.60 . The hTV-DR values were significantly higher than the Ct-DR values $(\mathrm{p}<0.001)$, suggesting that the tumor growth in these patients was more rapid before surgery (Table 1, Fig. 1).

We divided the patients' Ct-DR values into four categories: rather rapid growth, slow growth, stable, and decrease, using cutoffs at $0.5,0.1$, and -0.1 per year. In the 46 patients, the Ct-DR (/year) was > 0.5, 0.1-0.5, - 0.10.1 , and $<-0.1$ for $9,21,12$, and 4 patients, respectively (Table 2). The patients with sporadic MTC and those with hereditary MTC showed similar distributions of CtDR values $(p=0.923)$. Although these 46 patients had biochemically persistent disease, $26 \%$ of them showed stable disease and $9 \%$ of them showed a decrease in serum $\mathrm{Ct}$ values that may indicate shrinkage of their tumors. By definition, the hTV-DR should be larger than 0 . The hTV-DR (/year) values were $>1,0.5-0.1,0.1-0.5$, and $<0.1$ in $11,21,14$, and 0 patients, respectively (Table 3). The 18 patients with hereditary MTC had significantly higher hTV-DR values compared to the 28 patients with sporadic MTC $(p<0.001)$, possibly due to older age in sporadic MTC.

In the 28 patients with sporadic MTC, their Ct-DR values (/year) (median 0.17, range-0.42-5.70) were significantly lower than their hTV-DR values (/year) (median 0.50, range $0.26-1.10)(p=0.009)$ (Fig. 2). However, the Ct-DR values of four patients were higher than their hTV-DR values, suggesting that the preoperative tumor growth was slower than the postoperative growth, or the tumor arose much later than at birth in these patients. On the other hand, in all 18 of the patients with hereditary MTC, the Ct-DR values (/year) (median 0.21, range-1.25-1.90) were significantly lower than the hTV-DR values (/year) (median 1.21 , range $0.50-3.65)(p=0.001)$, suggesting there was

Table 2 Calcitonin doubling rates in 28 patient with sporadic MTC and 18 patients with hereditary MTC

\begin{tabular}{|c|c|c|c|c|c|c|c|}
\hline & $\mathbf{n}$ & $>0.5$ & & $0.1-0.5$ & -0.1 to 0.1 & $<-0.1$ & \\
\hline & & $\begin{array}{l}\text { Rather } \\
\text { growth }\end{array}$ & rapid & Slow growth & Stable & Decrease & \\
\hline Sporadic MTC & 28 & $5(18 \%)$ & & $13(46 \%)$ & $8(29 \%)$ & $2(7 \%)$ & \\
\hline Hereditary MTC & 18 & $4(22 \%)$ & & $8(44 \%)$ & $4(22 \%)$ & $2(11 \%)$ & * \\
\hline All patients & 46 & $9(20 \%)$ & & $21(46 \%)$ & $12(26 \%)$ & $4(9 \%)$ & \\
\hline
\end{tabular}

${ }^{*} p=0.923$. MTC; medullary thyroid carcinoma 
Table 3 Hypothetical tumor-volume doubling rates in 28 patient with sporadic MTC and 18 patients with hereditary MTC

\begin{tabular}{|c|c|c|c|c|c|}
\hline \multirow[t]{3}{*}{ 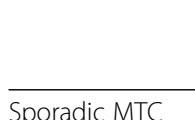 } & \multirow[t]{2}{*}{$n$} & \multirow{2}{*}{$\begin{array}{l}>1 \\
\text { Rapid growth }\end{array}$} & \multirow{2}{*}{$\begin{array}{l}0.5-1.0 \\
\text { Rather rapid growth }\end{array}$} & \multirow{2}{*}{$\begin{array}{l}0.1-0.5 \\
\text { Slow growth }\end{array}$} & \multirow{2}{*}{$\begin{array}{l}<0.1 \\
\text { Very slow }\end{array}$} \\
\hline & & & & & \\
\hline & 28 & $1(4 \%)$ & $13(46 \%)$ & 14 (50\%) & $0(0 \%)$ \\
\hline Hereditary MTC & 18 & $10(56 \%)$ & $8(44 \%)$ & $0(0 \%)$ & $0(0 \%)$ \\
\hline All patients & 46 & $11(24 \%)$ & $21(46 \%)$ & $14(30 \%)$ & $0(0 \%)$ \\
\hline
\end{tabular}

a more rapid tumor growth phase before surgery in all of these patients (Fig. 3).

\section{Discussion}

Collins et al. [6] reported that the growth of human tumors was exponential, and they proposed that the tumor growth rate is best described as tumor DT. Other research concerning the changes in serum $\mathrm{Ct}$ and carcinoembryonic antigen (CEA) values over time in patients with MTC who had persistent disease postoperatively and studies of the serum thyroglobulin values in patients with papillary thyroid carcinoma (PTC) after a total thyroidectomy has demonstrated that these values changed exponentially and that the DTs of these tumor marker values were very strong prognostic factors, supporting the concept described by Collins et al. [1, 7]. Thus, the DT is a well-validated parameter for the analyses and expressions of tumor growth.

However, the DT has two major limitations. First, if the volume of some of the tumors decreases over time, the tumors' DTs become negative values, which creates a discontinuity problem among positive and negative DT values. Second, the magnitude of the DT values is the opposite of the magnitude of the tumor growth rate. However, if the inverse of the DT is used (i.e., 1/DT), these limitations are resolved, and these inverse values should indicate the number of doublings that occur per unit of time. We thus proposed calling the 1/DT value the 'doubling rate' [3].
With the TV-DR we were able to demonstrate tumor volume kinetics of papillary microcarcinomas during active surveillance in individual patients [3]. Very interestingly, the volume of $17 \%$ of the tumors in that study decreased over time, showing negative TV-DRs values. Our calculation of the hypothetical TV-DR (an estimate of the lowest tumor growth rate) before presentation using the patient's age and tumor size at presentation showed that this value was much higher than the TVDR observed during active surveillance in most of the patients, suggesting that the tumor growth before presentation had been much more rapid [3]. We also observed that in patients with papillary thyroid microcarcinoma during active surveillance, the probability of tumor progression greatly decreased with age [8,9]. These data indicated that the spontaneous deceleration of tumor growth with age is a rather common phenomenon in papillary thyroid microcarcinomas.

To determine whether a similar phenomenon occurs in medullary thyroid carcinoma, we calculated the hTVDR applying our above-described hypothesis, and we also calculated the Ct-DR in the 46 patients with MTC showing biochemically persistent disease postoperatively. The estimated hTV-DRs were significantly higher than the observed Ct-DRs. This difference was more pronounced in the 18 patients with hereditary MTC than in the 28 patients with sporadic MTC. This thought experiment suggests that a rapid growth phase preceded surgery in the vast majority of the present patients with

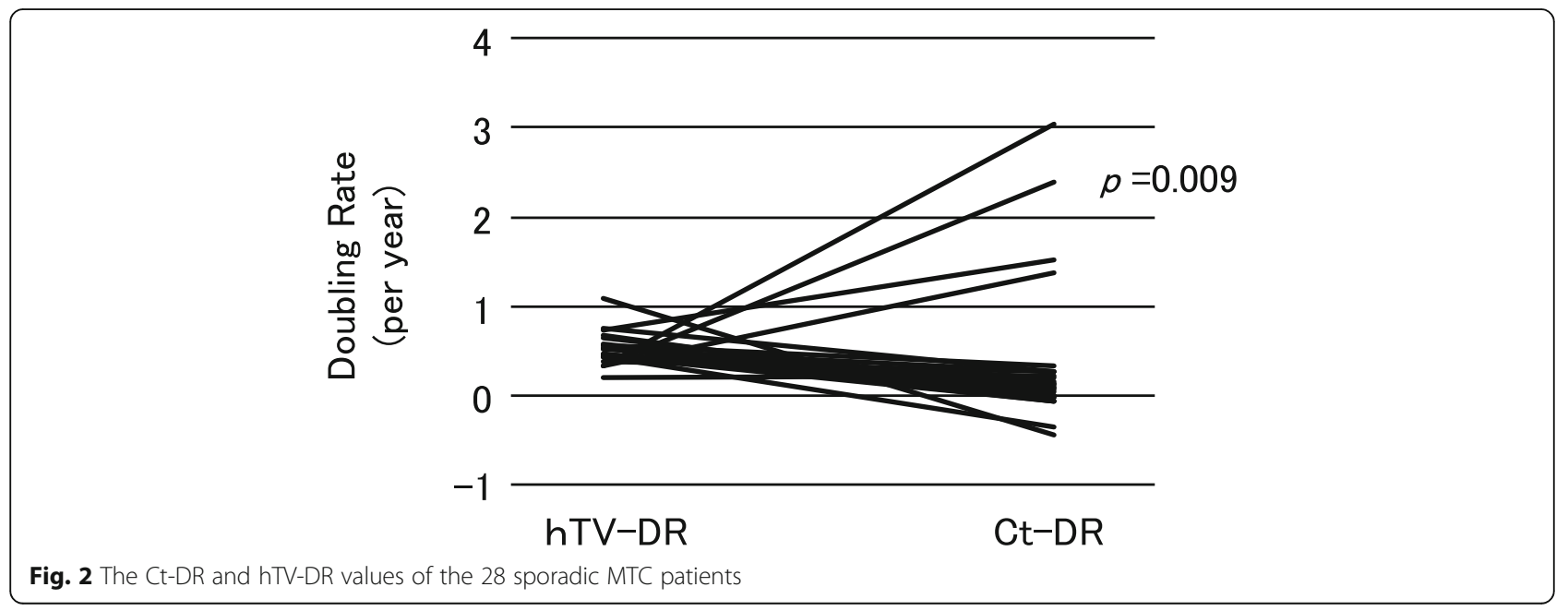




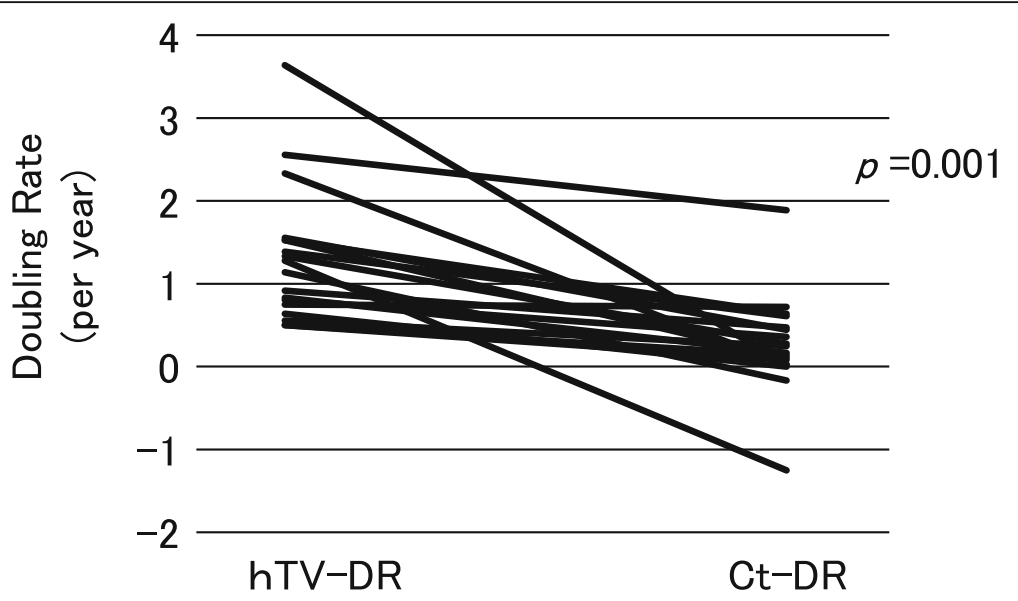

Fig. 3 The Ct-DR and hTV-DR values of the 18 hereditary MTC patients

MTC, especially in the 18 patients with hereditary MTC. Even in patients with sporadic MTC whose hTV-DRs were lower than those of hereditary MTC, possibly due to higher age of the former patients, their hTV-DRs were often higher than their Ct-DRs. These data thus suggest that most of the MTCs grew more rapidly before surgery. In other words, the growth rate of most of the MTCs was lower during follow-up than hypothetical maximal value during early stages of tumor development; the reason(s) for this deceleration remain to be clarified. This was especially evident for the hereditary MTCs. Hereditary MTCs arise at younger ages than sporadic MTCs [10-13], yet their prognosis is not worse than that of sporadic MTCs [13-15]. Four of the presented 28 patients with sporadic MTC had Ct-DR values that were higher than their hTV-DR values. This may be due to too small estimates of hTV-DR in these patients since they had large age values (old ages) at surgery. The actual origin of the tumor should have been much later than birth in these patients. Alternatively, the growth rate in these patients became more rapid after surgery.

Kasahara et al. reported a spontaneous decrease in serum thyroglobulin levels in pediatric patients with papillary thyroid carcinoma after total thyroidectomy [16]. We reported a spontaneous postoperative deceleration in the Ct-DT of patients with persistent hypercalcitoninemia in a long-term follow-up [17]. Ito et al. reported that the TV-DR values of the majority of papillary thyroid microcarcinomas decreased during the follow-up, after they showed increases in their tumor size during active surveillance [18]. These data suggest that a spontaneous deceleration of tumor growth following presentation or surgery is a common phenomenon in PTCs and MTCs as well. Of course, some of these tumors show a spontaneous aggressive change or an acceleration in their growth rate [17]. Acceleration in the tumor growth rate is typically seen in cases of poor differentiation and anaplastic change of differentiated thyroid carcinoma.

Our study has several limitations. The study design was retrospective, and the number of patients was small $(n=46)$ because we used only the data of a new $\mathrm{Ct}$ measurement method by ECLIA after April 2015. Second, in this thought-experiment study, we compared the Ct-DR with a hypothetical TV-DR. Although both values would reflect the tumor growth rate, the bases of their calculations are different. Since active surveillance for MTCs was not performed (unlike papillary thyroid microcarcinomas), our patients' TV-DR values after surgery based on the actual tumor size measurement were not available. However, our present findings provide some insight into the natural history of MTCs. Third, the number of hereditary patients was small at 18 and it was not possible to analyze according to $R E T$ mutations.

\section{Conclusions}

In the presented 46 patients with MTCs, the calculated hTV-DRs before surgery were significantly higher than the observed Ct-DRs after surgery, suggesting that there were rapid growth periods before surgery in the vast majority of the patients.

\section{Abbreviations \\ MTC: Medullary thyroid carcinoma; Ct: Calcitonin; DT: Doubling time; TV: Tumor volume; DR: Doubling rate; ATA: American Thyroid Association; ECLIA: Electrochemiluminescence immunoassay; CEA: Carcinoembryonic antigen; PTC: Papillary thyroid carcinoma}

\section{Acknowledgements}

Not applicable.

\section{Authors' contributions}

MK was involved in the study design, collection, analysis, interpretation of data and writing of the manuscript. AM was involved in the study design and writing the manuscript. All authors were involved in collection of data. All authors read and approved the final manuscript. 


\section{Funding}

Not applicable.

\section{Availability of data and materials}

All data is contained within the manuscript and additional files.

\section{Ethics approval and consent to participate}

This study was approved by the Ethics Committee in Kuma Hospital. Written informed consent for their case to be used was obtained from each patient.

\section{Consent for publication}

All authors provide consent for this publication.

\section{Competing interests}

The authors declare that they have no competing interests.

Received: 10 May 2020 Accepted: 8 July 2020

Published online: 21 July 2020

\section{References}

1. Miyauchi A, Onishi T, Morimoto S, Takai S, Matsuzuka F, Kuma K, et al. Relation of doubling time of plasma calcitonin levels to prognosis and recurrence of medullary thyroid carcinoma. Ann Surg. 1984;199:461-6.

2. Barbet J, Campion L, Kraeber-Bodéré F, Chatal JF, GTE Study Group. Prognostic impact of serum calcitonin and carcinoembryonic antigen doubling-times in patients with medullary thyroid carcinoma. J Clin Endocrinol Metab. 2005;90:6077-84

3. Miyauchi A, Kudo T, Ito Y, Oda H, Yamamoto M, Sasai H, et al. Natural history of papillary thyroid microcarcinoma: kinetic analyses on tumor volume during active surveillance and before presentation. Surgery. 2019; 165:25-30.

4. Wells SA Jr, Asa SL, Dralle H, Elisei R, Evans DB, Gagel RF, et al. American Thyroid Association guidelines task force on medullary thyroid carcinoma. Revised American Thyroid Association guidelines for the management of medullary thyroid carcinoma. Thyroid. 2015;25:567-610.

5. Sabra MM, Sherman EJ, Tuttle RM. Tumor volume doubling time of pulmonary metastases predicts overall survival and can guide the initiation of multikinase inhibitor therapy in patients with metastatic, follicular cellderived thyroid carcinoma. Cancer. 2017;123:2955-64.

6. Collins VP, Loeffler RK, Tivey H. Observations on growth rates of human tumors. Am J Roentgenol Radium Therapy, Nucl Med. 1956;76:988-1000.

7. Miyauchi A, Kudo T, Miya A, Kobayashi K, Ito Y, Takamura Y, et al. Prognostic impact of serum thyroglobulin doubling-time under thyrotropin suppression in patients with papillary thyroid carcinoma who underwent total thyroidectomy. Thyroid. 2011:21:707-16.

8. Miyauchi A, Kudo T, Ito Y, Oda H, Sasai H, Higashiyama T, et al. Estimation of the lifetime probability of disease progression of papillary microcarcinoma of the thyroid during active surveillance. Surgery. 2018;163:48-52.

9. Ito $Y$, Miyauchi A, Kihara M, Higashiyama T, Kobayashi K, Miya A. Patient age is significantly related to the progression of papillary microcarcinoma of the thyroid under observation. Thyroid. 2014;24:27-34

10. Moley JF, Fialkowski EA. Evidence-based approach to the management of sporadic medullary thyroid carcinoma. World J Surg. 2007;31:946-56.

11. van Heerden JA, Grant CS, Gharib H, Hay ID, Ilstrup DM. Long-term course of patients with persistent hypercalcitoninemia after apparent curative primary surgery for medullary thyroid carcinoma. Ann Surg. 1990:212:395-400.

12. Kihara M, Miyauchi A, Yoshioka K, Oda H, Nakayama A, Sasai H, et al. Germline RET mutation carriers in Japanese patients with apparently sporadic medullary thyroid carcinoma: a single institution experience. Auris Nasus Larynx. 2016:43:551-5.

13. Ito $Y$, Miyauchi A, Yabuta T, Fukushima M, Inoue $H$, Tomoda C, et al. Alternative surgical strategies and favorable outcomes in patients with medullary thyroid carcinoma in Japan: experience of a single institution. World J Surg. 2009;33:58-66.

14. Ito Y, Miyauchi A, Kihara M, Higashiiyama T, Fukushima M, Miya A. Static prognostic factors and appropriate surgical designs for patients with medullary thyroid carcinoma: the second report from a single-institution study in Japan. World J Surg. 2018;42:3954-66.

15. Jung KY, Kim SM, Yoo WS, Kim BW, Lee YS, Kim KW, et al. Postoperative biochemical remission of serum calcitonin is the best predictive factor for recurrence-free survival of medullary thyroid cancer: a large-scale retrospective analysis over 30 years. Clin Endocrinol. 2016:84:587-97.

16. Kasahara T, Miyauchi A, Kudo T, Nishihara E, Ito M, Ito Y, et al. Spontaneous slowing and regressing of tumor growth in childhood/adolescent papillary thyroid carcinomas suggested by the postoperative thyroglobulin-doubling time. J Thyroid Res. 2018;6470251.

17. Miyauchi A, Kudo T, Kihara M, Oda H, Ito Y, Miya A. Spontaneous deceleration and acceleration of growth rate in medullary thyroid carcinomas suggested by changes in calcitonin doubling times over longterm surveillance. World J Surg. 2019;43:504-12.

18. Ito Y, Miyauchi A, Kudo T, Higashiyama T, Masuoka H, Kihara M, et al. Kinetic analysis of growth activity in enlarging papillary thyroid microcarcinomas. Thyroid. 2019;29:1765-73.

\section{Publisher's Note}

Springer Nature remains neutral with regard to jurisdictional claims in published maps and institutional affiliations.
Ready to submit your research? Choose BMC and benefit from:

- fast, convenient online submission

- thorough peer review by experienced researchers in your field

- rapid publication on acceptance

- support for research data, including large and complex data types

- gold Open Access which fosters wider collaboration and increased citations

- maximum visibility for your research: over $100 \mathrm{M}$ website views per year

At BMC, research is always in progress.

Learn more biomedcentral.com/submissions 